\title{
Light concentration using hetero-junctions of anisotropic low permittivity metamaterials
}

\author{
Mohammad Memarian and George V Eleftheriades
}

Focusing incident power into an area of high concentration is of significant interest for various applications. In optics, this has been traditionally achieved with lenses where a higher curvature and lens permittivity typically result in shorter focal distances (Iow fID). In this work, we present designs and techniques for collecting, refracting and guiding incident light into an area of high power concentration (a hot spot) at extremely short distances. Specifically, a flat low-profile focusing mechanism is presented using a hetero-junction of anisotropic metamaterials (MTMs). The hetero-junction is formed from two cleaved finite slabs of low (near zero) permittivity anisotropic MTMs with rotated optical axes. The MTMs have near zero longitudinal permittivity while matched in the transverse direction. Such MTMs are shown to provide a unique ability to bend the transverse magnetic or p-polarized light away from the normal and along the interface, contrary to conventional dielectrics, and with minimal reflections; hence allowing for a low profile design. Realizations in the optical regime are presented using periodic bilayers of metal and dielectric. The proposed hetero-junction focusing device concentrates the normally incident plane wave and/or beam into a corresponding focal region similar to a lens via multiple refractions. The hetero-junction is capable of creating a hot spot very close to the device, much closer than dielectric lenses and it significantly outperforms the size requirements of thick high curvature lenses with low fID ratios. The proposed designs can find applications in various scenarios including solar and thermo photovoltaics, photodetectors, concentrated photovoltaics, non-imaging optics, micro- and nano-Fresnel lenses.

Light: Science \& Applications (2013) 2, e114; doi:10.1038/lsa.2013.70; published online 22 November 2013

Keywords: epsilon near zero; focusing; light concentration; metamaterials; non-imaging optics; photovoltaics

\section{INTRODUCTION}

In many electromagnetic and optical applications, it is desirable to collect light incident over a wide area and focus/concentrate it into a smaller area. Although the most notable application is imaging, other applications include non-imaging and illumination optics, ${ }^{1}$ concentrated photovoltaics ${ }^{1-3}$ and electromagnetic concentrators ${ }^{4-7}$ to name a few. In all these scenarios, the collected electromagnetic waves are refracted and guided to create an area of high power concentration. Various solutions have been proposed so far, such as using traditional lenses, ${ }^{1,2}$ Fresnel lenses ${ }^{1,3}$ and transformation optics devices, ${ }^{4,7,8}$ depending on the application. A potential solution for light concentration is using the growing field of metamaterials.

Metamaterials (MTMs), materials with uncommon constitutive parameters, have enabled new frontiers and various scientific breakthroughs such as the 'superlens' for perfect imaging ${ }^{9}$ and the electromagnetic 'cloaking' ${ }^{8}$ in the past decade or so. Such engineered structures have yielded an 'effective' electromagnetic response that is normally not encountered in nature. Aside from enabling these new frontiers and interesting scientific properties such as a negative index of refraction, ${ }^{10,11}$ negative refraction, ${ }^{12}$ indefinite media ${ }^{13}$ or nearfield imaging, ${ }^{11,14}$ MTMs have shown to be beneficial for various existing applications. For instance, MTMs show superior performance over prior techniques for absorption, wave guidance and narrowband emission of light for solar and/or thermal photovoltaic cell applications. Recently, various works have used metamaterials for the design of thermal absorbers ${ }^{15-20}$ and selective thermal emitters $^{21-26}$ potentially for thermal photovoltaics (TPV), as well as Solar-TPV systems. ${ }^{27,28}$

A certain class of metamaterials have permittivity values close to zero which are also known as epsilon near zero (ENZ) materials in the literature. ${ }^{29-31}$ They have shown interesting usages, e.g., in tunneling energy through bends and channels ${ }^{30}$ and tailoring the radiation phase pattern. ${ }^{31}$ Although isotropic bulk ENZs are hard to achieve, anisotropic MTMs can be realized using subwavelength periodic bilayers of two materials, ${ }^{32}$ such as bilayers of a metal and dielectric, enabling negative refraction in the optical and infrared regimes. ${ }^{33,34}$ Such layered structures can be tailored to achieve near zero permittivity at least along one axis, which has enabled the realization of the hyperlens with hyperbolic dispersion MTMs. ${ }^{35-38}$

In this work, we propose a flat low-profile device based on a heterojunction of anisotropic ENZ metamaterials, which leads to focusing and power concentration of the incoming light. The anisotropic MTMs have low longitudinal permittivity and are matched in the transverse direction for minimal reflections. The proposed device is

The Edward S. Rogers Sr. Department of Electrical and Computer Engineering, University of Toronto, Toronto, Ont. M5S 2E4, Canada

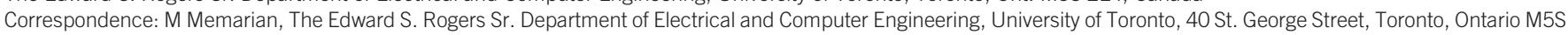

2E4, Canada

E-mail: m.memarian@mail.utoronto.ca

Received 2 June 2013; revised 9 August 2013; accepted 14 August 2013 
low profile and flat and concentrates the field much like a lens does into a spot. In contrast to other designs, where the field intensity is increased inside a transformed medium, ${ }^{4-7}$ this design focuses the power into a region outside in the host medium (e.g., air). It is shown that such a device can in fact outperform thick lenses with very low $f / D$ ratios, to create areas of high power concentration very close to the exit facet of the device, i.e., at short focal distances. It also outperforms the recently reported flat and thin lenses realized using arrays of optical antennas $^{39,40}$ in terms of their $f / D$ ratios. The proposed focusing hetero-junction device is an alternative method for extremely close power concentration under normal incidence.

We demonstrate how to logically construct such a focusing device out of ENZ hetero-junctions, by exploring the unique refraction properties of anisotropic ENZs based on ray optics and corresponding dispersion diagram analysis. Unlike transformation optics ${ }^{8}$ which typically results in very complex media that are hard to realize in practice, this approach yields simple uniaxial structures that are easier to realize in the optical regime.

\section{MATERIALS AND METHODS}

Our uniaxial MTM media of interest are assumed to be non-magnetic with $\mu_{\mathrm{r}}=1$ and have a permittivity tensor

$$
\overline{\bar{\epsilon}}=\left(\begin{array}{ccc}
\epsilon_{x} & 0 & 0 \\
0 & \epsilon_{y} & 0 \\
0 & 0 & \epsilon_{z}
\end{array}\right) \epsilon_{0}
$$

For an anisotropic uniaxial medium $\left(\epsilon_{y}=\left\{\epsilon_{x}\right.\right.$ or $\left.\left.\epsilon_{z}\right\}, \epsilon_{x} \neq \epsilon_{z}\right)$, transverse magnetic (TM)-polarized light with an in-plane electric field in the $x-z$ plane experiences different permittivity values along the $z$ and $x$ axes.

\section{Bending light away from normal}

Consider a TM plane wave that is incident at a slightly off-normal angle on the interface of air and an arbitrary medium, as shown in the $x-z$ plane of incidence in Figure 1a. If the medium is a typical dielectric such as glass, according to Snell's law, the wave refracts such that it always makes a smaller angle with the normal as shown in Figure 1b,
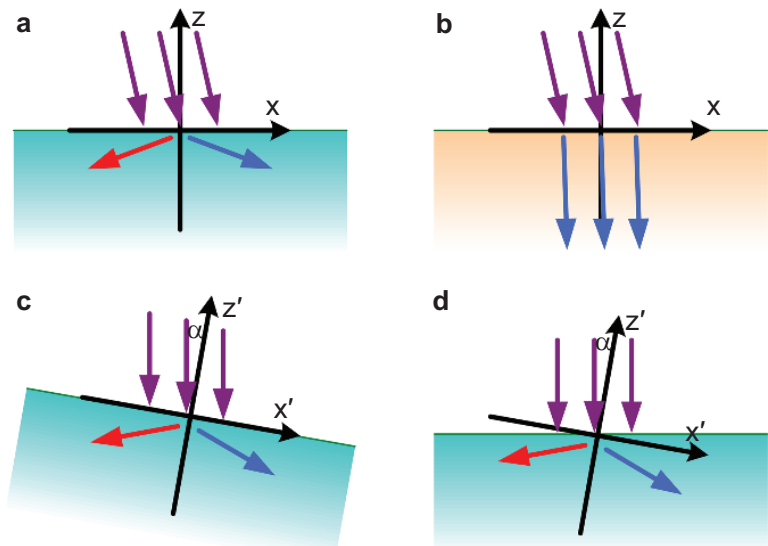

Figure 1 (a) Off-normal light incident on the interface of air and a half-space of a material filled with an anisotropic MTM with low longitudinal and matched transverse permittivity values $\left\{\epsilon_{z} \rightarrow 0, \epsilon_{x}=1\right\}$ causing refraction away from normal. Both positive (blue) and negative (red) refraction is possible depending on the choice of MTM. (b) Interface with a regular isotropic dielectric $\epsilon_{r}>1$ which always positively refracts light closer to the normal. (c) A rotated MTM with an inclined interface and (d) a rotated MTM cleaved surface along $x$-axis, to refract the $z$ directed incident light away from the $z$-axis. MTM, metamaterial. since glass is a dense medium $\left(\epsilon>\epsilon_{0}\right)$ compared to air. But if the medium has low permittivity $\left(\epsilon<\epsilon_{0}\right)$, the off-normally incident light refracts differently, such that it makes a larger angle away from the normal to the interface. In the case of a zero permittivity MTM $(\epsilon \rightarrow 0)$, the refracted wave flows almost parallel to the interface of the MTM and air, as shown in Figure 1a. This seemingly simple refraction is not encountered in nature and is not typically noted. It is, however, of significant importance and will be used for the operation of the light concentrator.

An isotropic zero permittivity MTM is typically not realizable in nature and it is also mismatched to air (impedance $=\sqrt{\mu / \epsilon}$ ). The latter issue causes reflections for the incident light at the interface of the MTM reducing the transmitted power. We find, however, that in order to achieve the desired refraction, the MTM does need to be isotropic. The condition for refracting the wave away from the normal is to have a close to zero longitudinal permittivity (e.g., $\epsilon_{z} \rightarrow 0$ ). The permittivity in the transverse direction does not need to be low and can in fact be used to match the MTM to air for normally incident light. Deriving the reflection coefficient at the interface by applying the boundary condition for the continuity of the tangential electric and magnetic fields, it is found that for an arbitrary angle of incidence $\phi$, the reflection coefficient is

$$
r=\frac{\cos \phi \sqrt{\epsilon_{x}}-\sqrt{1-\sin ^{2} \phi / \epsilon_{z}}}{\cos \phi \sqrt{\epsilon_{x}}+\sqrt{1-\sin ^{2} \phi / \epsilon_{z}}}
$$

This shows that for normal incidence $\phi=0^{\circ}, \epsilon_{x}=1$ results in zero reflections.

Therefore, we arrive at an anisotropic MTM with low longitudinal $\epsilon_{z} \rightarrow 0^{ \pm}$and matched transverse $\epsilon_{x}=1$ permittivity values as the optimum solution for our refraction of interest. Incidentally this is the opposite of the operation of the hyperlens ${ }^{35}$ where the transverse permittivity was close to zero. As noted, there are two possible solutions depending on the sign of $\epsilon_{z}$. Figure la shows the power flow (Poynting vector) for the two possibilities corresponding to positive (blue) and negative (red) refraction. From a dispersion standpoint, this means that the MTM follows either elliptic or hyperbolic dispersion, respectively.

The same anisotropic medium can be used to refract a completely $z$ directed incident light away from the $z$-axis. This is done by rotating the crystal by a slight angle $\alpha$ in the $x-z$ plane as shown in Figure 1c, where the rotation causes an inclined surface. The permittivity tensor of such rotated crystal can be found by a coordinate transformation and using a rotation matrix $\overline{\bar{R}}(\alpha)$, where

$$
\overline{\bar{R}}(\alpha)=\left(\begin{array}{ccc}
\cos \alpha & 0 & -\sin \alpha \\
0 & 0 & 0 \\
\sin \alpha & 0 & \cos \alpha
\end{array}\right)
$$

The final permittivity matrix of the rotated slab $\overline{\bar{\epsilon}}$ in the $x-z$ coordinates is $\overline{\bar{\epsilon}}=\overline{\bar{R}}(-\alpha) \overline{\bar{\epsilon}}^{\prime} \overline{\bar{R}}(\alpha)$. The same rotated crystal may also be cleaved along the $x$-axis. The flat surface will still bend the normal incident light with a large angle away from the normal, making it propagate along the interface as shown in Figure 1d.

We can therefore bend the direction of power flow of normally (as well as off-normally) incident light farther away from the normal to the interface. This refraction with minimal reflections is not possible with other choices of values for the permittivity tensor (e.g., it is not encountered in nature with typical dielectrics) and is a direct consequence of using an anisotropic low permittivity medium. This 
refracted wave is a free propagating wave in the lower half-space and is not a 'bound surface wave', and thus can be further guided if needed. Our intention is not to couple to surface waves bounded to the interface as they do not carry real power when leaving the interface.

The refraction capability suggests that one can create a longer transverse path length for the refracted wave and a much shorter longitudinal path. It therefore suggests that one can use a thin material, as the longitudinal component of the ray path is much shorter than the transverse. This has a direct consequence for realizing low profile focusing devices as shown in this work. Another consequence of this result is that it can potentially allow thinner absorbers (say, for TPV applications), by increasing the chance of absorption of the incoming power in the transverse direction despite its small thickness.

\section{Physical realization}

The desired anisotropic low permittivity MTMs can be realized with layers of metal and dielectric at optical frequencies. It has been long known that subwavelength periodic bilayers of materials can be effectively treated as a homogenous medium with an effective uniaxial birefringent permittivity tensor. ${ }^{32}$ This has been used in various stu$\operatorname{dies}^{33}$ and is particularly useful for the realization of ENZ MTMs ${ }^{29}$ where the desired 'close to zero permittivity' is typically achieved by interleaving subwavelength layers of a material with positive permittivity and a material with negative permittivity, such that the effective medium is zero permittivity. ${ }^{35-38}$ The effective permittivity values along the optical axis and normal to the optical axis of a layered structure using the first-order Effective Medium Theory formulas are: ${ }^{32,38}$

a
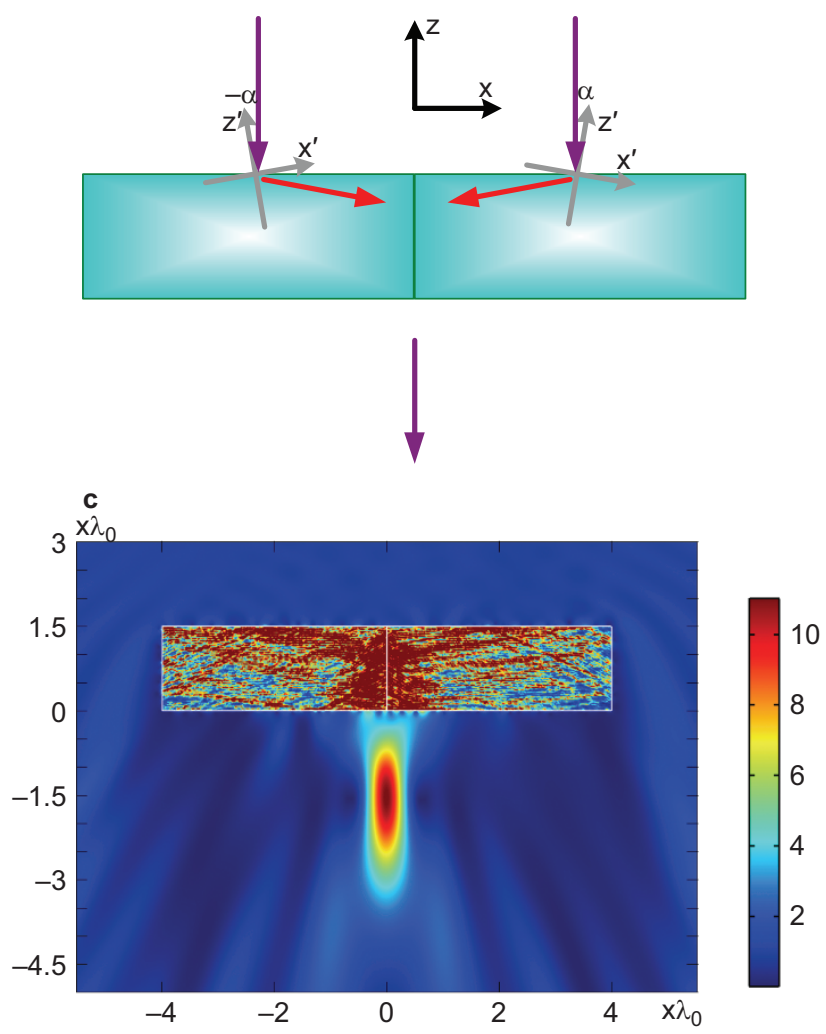

$$
\begin{gathered}
\epsilon_{\mathrm{axis}}=\frac{\epsilon_{m} \epsilon_{d}}{(1-p) \epsilon_{m}+p \epsilon_{d}} \\
\epsilon_{\perp \text { axis }}=p \epsilon_{m}+(1-p) \epsilon_{d}
\end{gathered}
$$

where $\epsilon_{m}$ and $\epsilon_{d}$ are the permittivity values of the metal and dielectric layers, respectively, $p$ is the filling ratio of the metal layer (thickness of the metal layer divided by the sum of the thickness of metal and dielectric layers) and the optical axis is normal to the layers.

Depending on the operating wavelength and desired level of loss, one may choose a variety of materials for realizing the required anisotropy. For instance for the low-loss focusing device presented here we utilize bilayers of silver (Ag) having $\epsilon_{m}=-18+0.5 j^{35}$ for the metal layer (loss and dispersion accounted for) and glass $\epsilon_{d}=2.2$ for the dielectric layer, at a free-space operating wavelength of $\lambda_{0}=633 \mathrm{~nm}$.

\section{RESULTS AND DISCUSSION}

\section{Light concentration using MTM hetero-junctions}

Figure 2a proposes the MTM based focusing device that collects light over a large area and concentrates it into a hot spot. The device comprises a hetero-junction of two anisotropic low permittivity MTMs $\left\{\epsilon_{z^{\prime}} \rightarrow 0, \epsilon_{x^{\prime}}=1\right\}$ placed side by side. Each MTM is rotated by a small angle $( \pm \alpha)$ giving rise to oppositely rotated principal axes on the two sides of the junction. The MTMs are finite slabs and their interfaces are cleaved along the $x$-axis.

According to the operation discussed in Figure 1d, the normally incident light bends away from the normal on each side of the

b
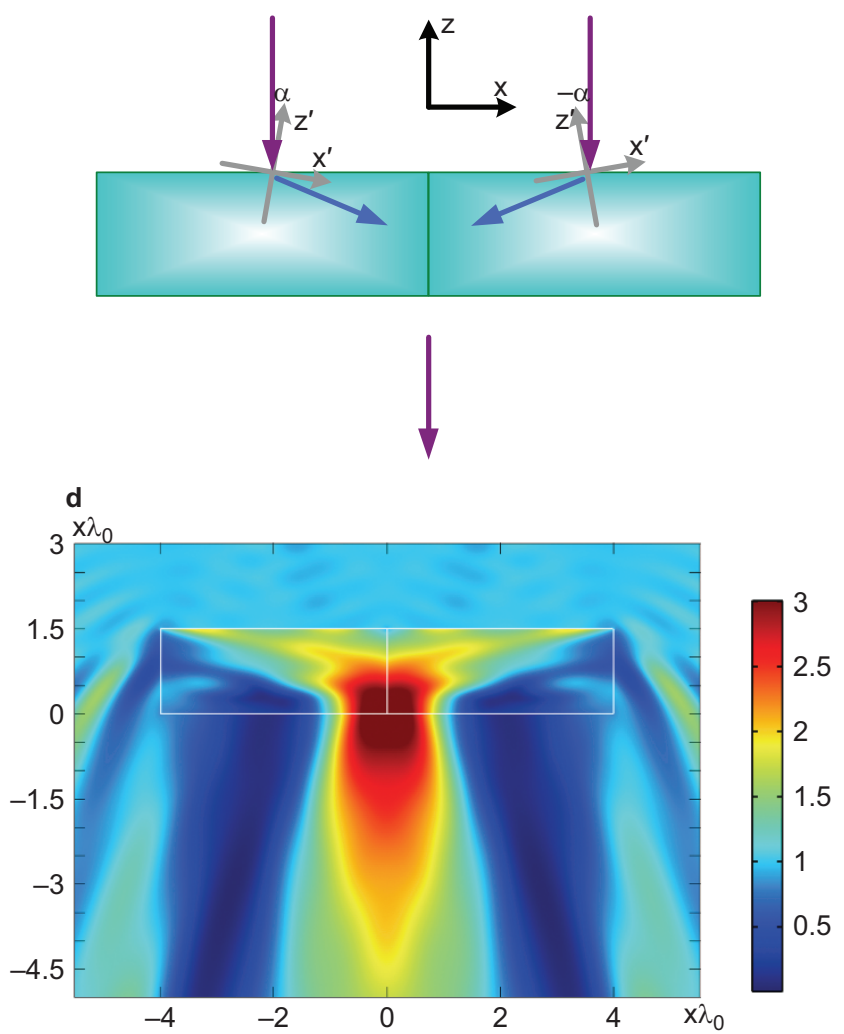

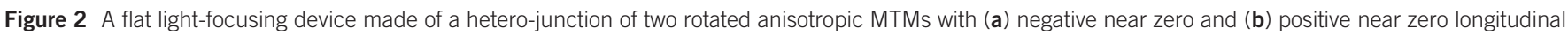

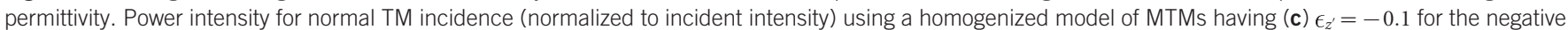
refracting device and $(\mathbf{d}) \epsilon_{z^{\prime}}=+0.1$ for the positive refracting device and $\epsilon_{x^{\prime}}=1, \alpha= \pm 10^{\circ}$. MTM, metamaterial; TM, transverse magnetic. 
hetero-junction. The MTMs are rotated such that the waves are refracted accordingly on each side such that power is forced to flow towards the center of the structure. The light is then transmitted through to create a spot of high field concentration on the other side of the structure in air. Hence, the overall hetero-junction operates similarly to a lens under normal incidence and focuses light into a spot. The device shown in Figure 2a uses negative refraction with $\epsilon_{z^{\prime}} \rightarrow 0^{-}$. A similar device using positive refraction with $\epsilon_{z^{\prime}} \rightarrow 0^{+}$can also be envisioned as shown in Figure 2b. As shown, the MTMs in Figure $2 \mathrm{~b}$ must be rotated opposite to that of Figure $2 \mathrm{a}$, such that the refraction again forces the power towards the center.

Figure $2 \mathrm{c}$ shows fullwave simulation results of the field magnitude for a device with parameters $\left\{\epsilon_{z^{\prime}}=-0.1, \epsilon_{x^{\prime}}=1\right\}$, i.e., the case of Figure $2 \mathrm{a}$ having MTMs with hyperbolic dispersion. The simulation results of a device realized with elliptical dispersion MTMs $\left\{\epsilon_{z^{\prime}}=+0.1, \epsilon_{x^{\prime}}=1\right\}$ is shown in Figure 2d. The tilt angle of the MTMs for both devices is $\alpha= \pm 10^{\circ}$. It can be clearly seen that an area of high field concentration or hot spot is indeed achieved in both cases close to the bottom interface. It should be noted that the top interface of the devices does not necessarily need to be cleaved and can be left at the corresponding slanted angles as was done in Figure 1c. However, a cleaved surface along the $x$-axis allows realizing a more flat and low profile design.

\section{Theory of operation of the light concentrator}

The exact operation of the negative refracting device in Figure 2a can be best understood using the equifrequency contours shown in Figure 3a. The wave vector/phase velocity and the power flow are traced out throughout the structure and outside. The diagram is constructed based on the phase matching boundary condition at each interface, i.e., the tangential wave vector must be continuous. The direction of the Poynting vector (power flow) as well as the group velocity is normal to the equifrequency contour. The propagation in the anisotropic medium prior to rotation is governed by

$$
\frac{k_{x^{\prime}}^{2}}{\epsilon_{z^{\prime}}}+\frac{k_{z^{\prime}}^{2}}{\epsilon_{x^{\prime}}}=k_{0}^{2}
$$

The figure shows the propagation of a TM wave incident on the MTM on the right side of the hetero-junction. The normal incident

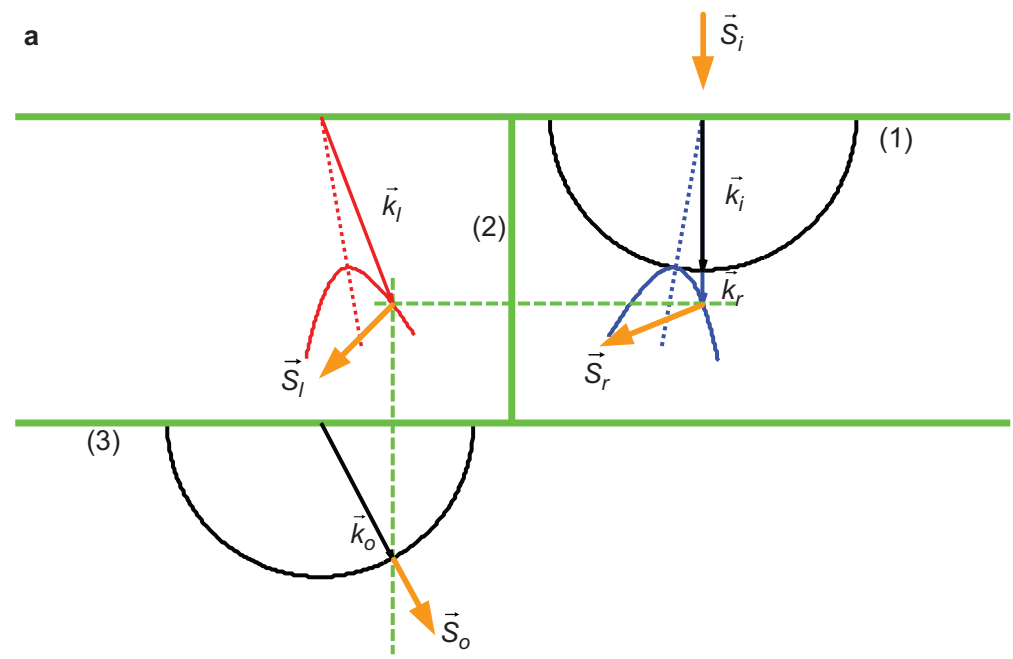

b

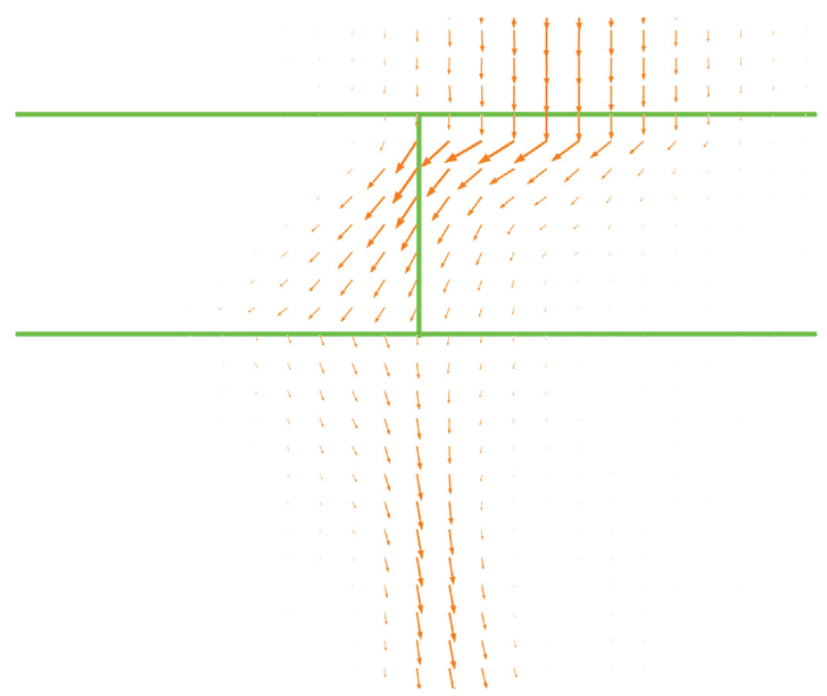

C

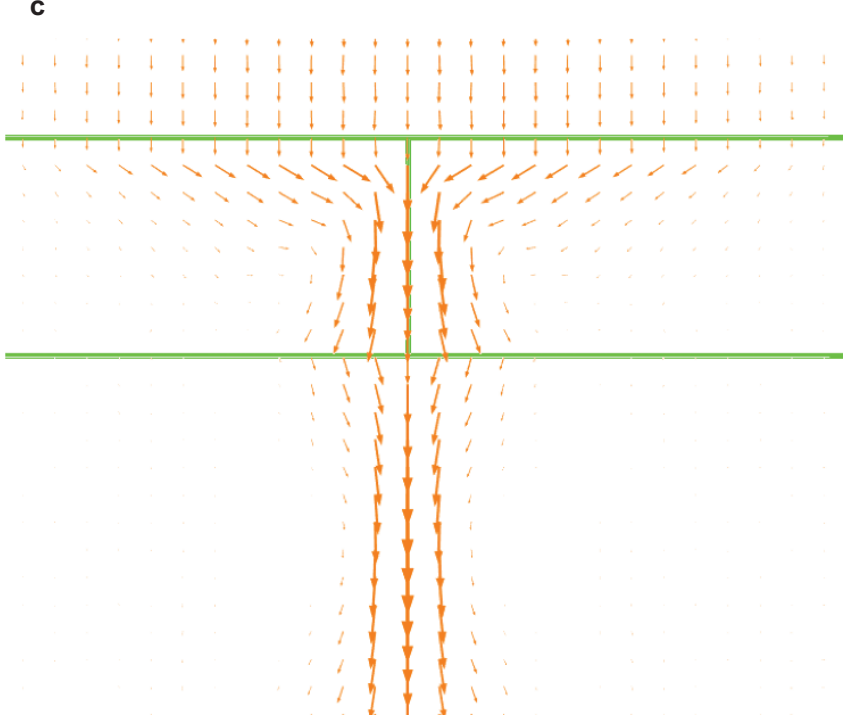

Figure 3 (a) Dispersion diagram showing wave vector and refraction at different boundaries, equifrequency contours inside hyperbolic media, as well as predicted directions of Poynting vector. Fullwave simulations of the power flow (Poynting vector) for (b) partial illumination and (c) full illumination of the device. 
wave $\vec{k}_{i}$ impinging on the air/MTM interface (1), phase matches to a wave vector $\vec{k}_{r}$ in the right-side MTM that also has a zero tangential wave vector. Because of the crystal rotation, the equi-frequency contour of the right-side MTM is tilted by the angle $\alpha$ causing $\vec{k}_{r}$ to be larger than $\vec{k}_{i}$. The normal to the equifrequency contour at this point defines the direction of the Poynting vector $\vec{S}_{r}$, which as mentioned before is bent along the interface, causing power to flow away from the normal and to the left. Due to the choice of the permittivity tensor, the dispersion in both MTMs is hyperbolic leading to negative refraction at interface (1) and is consistent with the designated power flow. The negatively refracted wave in the right-side MTM travels towards the center along $\vec{S}_{r}$, while its phase changes along the wave vector $\vec{k}_{r}$. At the interface (2), $\vec{k}_{r}$ is completely tangential to the interface and therefore phase matches a wave $\vec{k}_{l}$ in the left side MTM as shown. In order to maintain causality, the power now must flow away from interface (2) and therefore, we need to choose the appropriate branch of the hyperbola in the left-side MTM. The direction of power flow changes to $\vec{S}_{l}$, due to the $-\alpha$ tilt of the optical axis in the left side MTM and the normal to the contour at that point. The wave in the left-side MTM finally experiences another negative refraction at the MTM/air interface (3). Again the tangential wave vector at the interface is continuous, leading to the output wave $\vec{k}_{o}$. The output power flow in air $\vec{S}_{o}$ is now in the $+x$ direction opposite to the flow in the MTM and is directed towards the center.
Figure $3 \mathrm{~b}$ shows the power flow (Poynting vector) for a Gaussian beam incident on the right side of the device, using full-wave simulation. The simulated power flow confirms the predicted behavior from theory in Figure 3a. The figure confirms that the power incident on the right-side MTM is refracted towards the middle, then refracted into the left-side MTM and is then negatively refracted into air towards the center at the bottom side.

If the power was only incident on the left-side MTM, a similar behavior could be traced out for the transmitted and internal waves, only mirrored about the $z$-axis. In the final case where the incident wave illuminates both sides of the device, the resulting transmitted wave is therefore the superposition of the output from both sides. The two transmitted power flows concentrate towards the middle, creating a high concentration focal spot along the $z$-axis on the other side of the structure. Such focusing action is shown in the simulation results of the power flow in Figure 3c.

\section{Bilayered negatively refracting focusing device}

The fullwave simulation results of an actual focusing device realized with the Ag/glass bilayer implementation are shown in Figure $4 \mathrm{a}$ and $4 \mathrm{~b}$. The power density in Figure 4a is again normalized to the power density of the normally incident plane wave illuminating the structure. It can be clearly seen that a distinct bright focus point is achieved, with significant field enhancement at that point, similar to the results of the

a

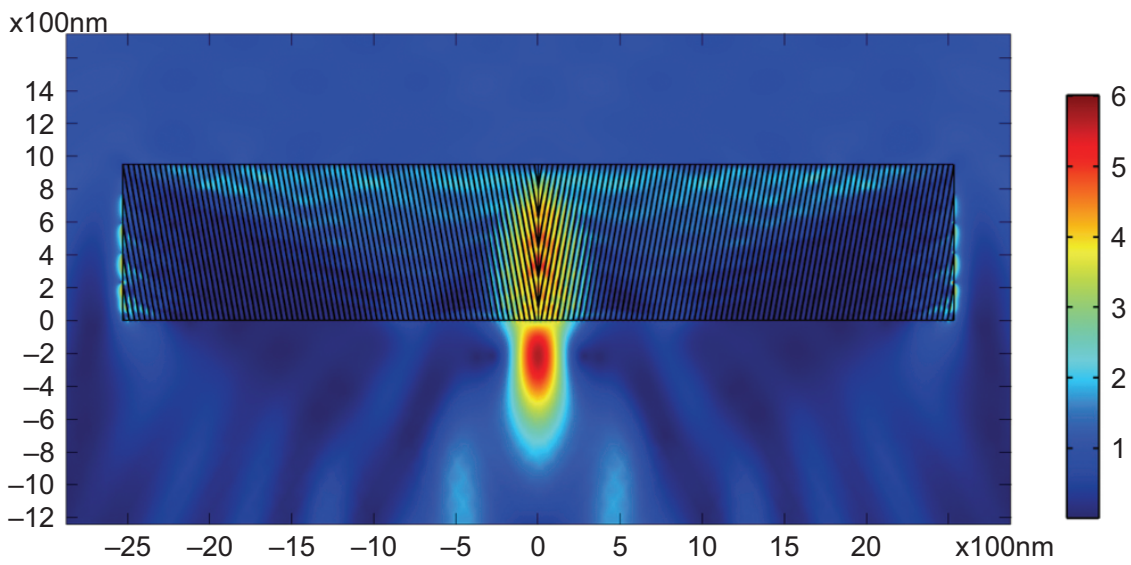

b

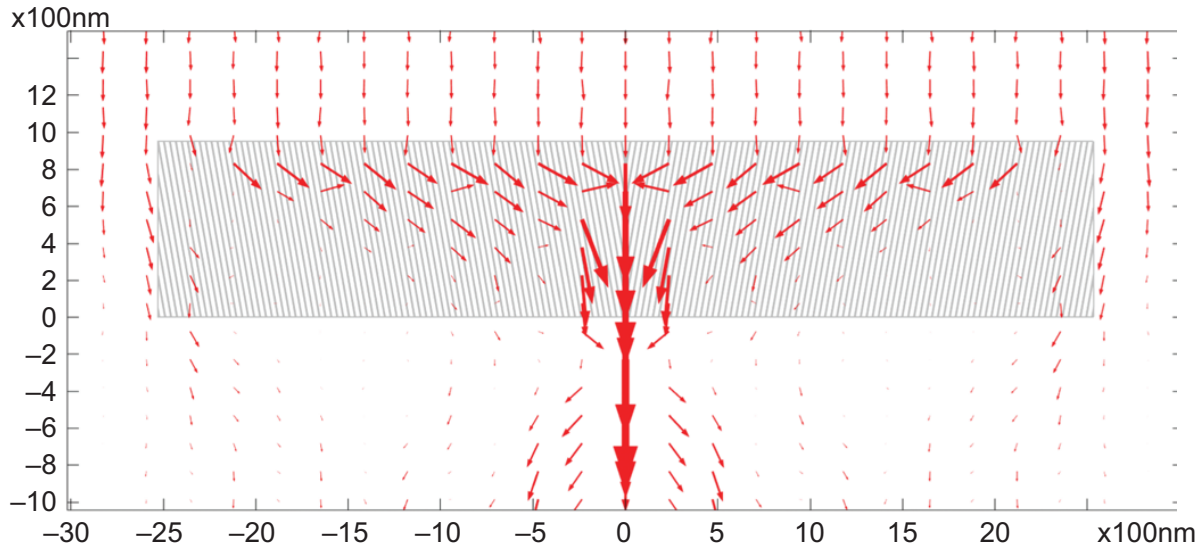

Figure 4 (a) Power density normalized to the incident power density for the focusing hetero-junction device realized with layers of Ag and glass at $\lambda_{0}=633 \mathrm{~nm}$, under normal plane wave illumination. (b) Power flow (Poynting vector) throughout the device showing concentration towards the middle. 
ideal case of the negatively refracting focusing device in Figure $2 c$. This demonstrates a focusing device with realistic materials and having losses included, operating at $\lambda_{0}=633 \mathrm{~nm}$. The power flow through this layered focusing device is also shown in Figure $4 \mathrm{~b}$. It can be clearly seen that the focusing device collects and bends the incident normal power at the top surface and towards the middle via refraction, resulting in a high concentration of transmitted power at the middle of the structure. The MTMs of the focusing device are implemented with the $\mathrm{Ag} /$ glass bilayer combination with a period of $L=\lambda_{0} / 10$, filling ratio of $p=0.126$ and tilt angle of $\alpha= \pm 10^{\circ}$. Although this device has an effective transverse permittivity of $\epsilon_{x} \approx 2.5$ due to the choice of materials, it is still far better matched to air than the corresponding isotropic low permittivity medium $\left(\epsilon_{x}=\epsilon_{z} \rightarrow 0\right)$, according to the expression for reflection ' $r$ ' presented earlier. Note that the amount of the reflected power is related to $|r|^{2}$.

To our knowledge, this is the first time that a flat low-profile concentrator of light has been devised with the use of anisotropic MTMs. Also, in contrast to other designs where the field intensity is increased inside a medium, ${ }^{4-6}$ this design focuses the power in the host medium (e.g., air) similar to a lens. This is particularly useful for solar-concentration applications, where light with high concentration needs to shine the PV and can be used as a non-imaging concentrator in focusing power for concentrated photovoltaics applications. ${ }^{1}$

In general, the proposed light collecting hetero-junction structure may be used in two different types of applications. The first set of applications is when it operates as a focusing mechanism to transmit and concentrate the power into a hot spot in air as shown in Figure 2. This hetero-junction operates as a compact and low profile focusing device with small dimensions on the order of several wavelengths at the operating frequency. Various scenarios such as concentrated photovoltaics applications or microlenses can benefit from this configuration. Such scenarios would require maximum transmission of field to the hot spot and therefore, the device should be designed for low losses. Although we suggest here a layered realization with Ag and glass, other metal/dielectric combinations may also offer advantageous low loss operation, as well as the more recently explored low-loss plasmonic metamaterials, ${ }^{41}$ depending on the wavelength of operation.

In a second type of applications, the same structure can be designed to be highly lossy, in order to be used as a thin absorber (or equivalently a narrow band and narrow angle emitter) applicable to solar and TPV applications, if tuned to the desired wavelength. In this scenario, the transmission of the field is not of importance and the effort is to make a long transverse path for the rays inside the structure in order to increase the chance of absorption. It can create an area of high concentration in the middle of the structure such that this power is absorbed in order to heat up the structure itself and/or a secondary structure in contact with the hetero-junction. Even a single rotated slab of the proposed device may also be used as the thin absorber as it yields a well matched medium that can absorb the incident power with a thin depth.

\section{Short focal distance}

The proposed focusing device can achieve a focal point very close to the exit facet of the device, closer than bulky lenses with extremely low flD ratios. Typically, short focal distances are achieved with a thick lens having a high radius of curvature, or even elliptical shapes. Not only elliptical designs are harder to make, but such large designs may not be always feasible, especially if the lens is a microlens with a small size in the order of several wavelengths and/or in applications where a low profile is desired.
The close focusing ability of the device can be better seen in the comparison study summarized in Figure 5. We compare the locations of the focal points for a plano-convex, a convex-convex lens and a hyperhemispherical lens all made with glass $\epsilon_{r}=2.5$, with the proposed ideal hetero-junction focusing device with parameters $\left\{\epsilon_{z^{\prime}}=-0.3, \epsilon_{x^{\prime}}=1, \alpha= \pm 10^{\circ}\right\}$. The aperture size and thickness (bounding dimensions) of the first two lenses are kept equal to the focusing device $\left(8 \lambda_{0} \times 2 \lambda_{0}\right)$, such that the performance of the three scenarios are evaluated under the same size requirements. In order to achieve the closest focus, the two lenses must have the maximum radius of curvature that fits this bounding dimension. Therefore the lenses have zero thickness on the sides to a maximum thickness of $2 \lambda_{0}$ in the middle. All scenarios are illuminated with a Gaussian beam of waist size $\omega_{0}=6 \lambda_{0}$, and the power is normalized to the peak power of the incident beam in free space.

The simulation results in Figure $5 \mathrm{~b}$ and $5 \mathrm{c}$ demonstrate that the distance of the peak of the focal area from the exit face is $3.5 \mu \mathrm{m}=5.53 \lambda_{0}$ for the convex lens and $4 \mu \mathrm{m}=6.3 \lambda_{0}$ for the planoconvex lens, respectively. However, the proposed hetero-junction focusing device in Figure 5a develops a focus at $0.388 \mu \mathrm{m}=0.61 \lambda_{0}$, a very short focal distance given the allowed dimensions. This shows a considerable improvement over traditional lenses of the same size in achieving close by focal points using the proposed structure, for the same aperture and thickness dimensions.

Figure $5 \mathrm{~d}$ shows a thick hyperhemispherical glass lens of the same aperture size $\left(2 R=8 \lambda_{0}\right)$ and a thickness of $R\left(1+1 / \sqrt{\epsilon_{r}}\right)=$ $4.13 \mu \mathrm{m}=6.53 \lambda_{0}$, according to Filipovic et al ${ }^{42}$ Such a thick lens provides a focal distance of $0.9 \mu \mathrm{m}=1.42 \lambda_{0}$, which is still longer than the focal length achieved with the proposed device. A hyperhemispherical lens with the same aperture size and made of quartz $\left(\epsilon_{r}=3.8\right)$ has a focal distance roughly equal to the proposed focusing device but is considerably thicker at $6.05 \lambda_{0}$. This demonstrates that a high permittivity lens of very thick size with hyperhemispherical (or elliptical) design is required to achieve the same focal distance, showing the limitation of traditional lenses in achieving focal spots very close to the lens. Another point of interest is that without curvature, a simple dielectric cannot provide focusing, whereas here the proposed design provides a flat structure without curvature that yields better results than lenses.

From the color plots of Figure $5 \mathrm{a}-5 \mathrm{c}$, it is evident that the proposed device develops a focal spot that is much closer and also stronger than the focus of the traditional lens of the same dimensions. Of course an actual lossy implementation of the device can reduce the strength of the fields at the focal spot slightly depending on the materials and implementation. This is mainly due to the losses that the ray paths exhibit by traveling through the lossy device. However, even a lossy device outperforms the corresponding lens by developing a very closeby focus that is actually still stronger than the lens's focal strength. For instance, consider an actual lossy focusing device made with bilayers of $\mathrm{Ag} /$ glass similar to Figure 4 and with dimensions $\left(8 \lambda_{0} \times 1.5 \lambda_{0}\right)$. If tuned for a short focal distance, the peak power of the focus is about 1.25 of the peak power of the lossless convex-convex lens of the same bounding dimensions. This lossy focusing device has an extremely short focal distance of only $0.175 \mu \mathrm{m}=0.277 \lambda_{0}$ away from the device, while the corresponding convex lens has a focal distance of $4.75 \mu \mathrm{m}=7.5 \lambda_{0}$. This is an extremely short focal distance resulting in an unprecedented $\mathrm{fl}$ $D=0.034$ at $\lambda_{0}=633 \mathrm{~nm}$. This result even significantly outperforms (by an order of magnitude) the recently demonstrated extreme short focusing using transmitarrays of optical antennas, ${ }^{39,40}$ which reported a best case of $f / D=0.625(2.5 \mu \mathrm{m} / 4 \mu \mathrm{m})$ at $\lambda_{0}=676 \mathrm{~nm}^{40}$ 
a

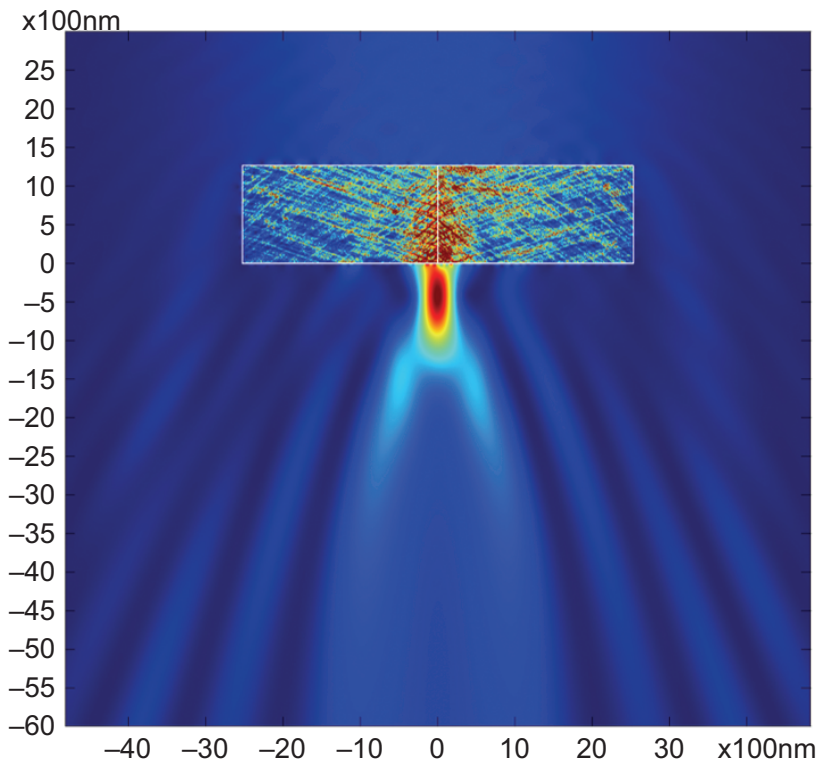

c

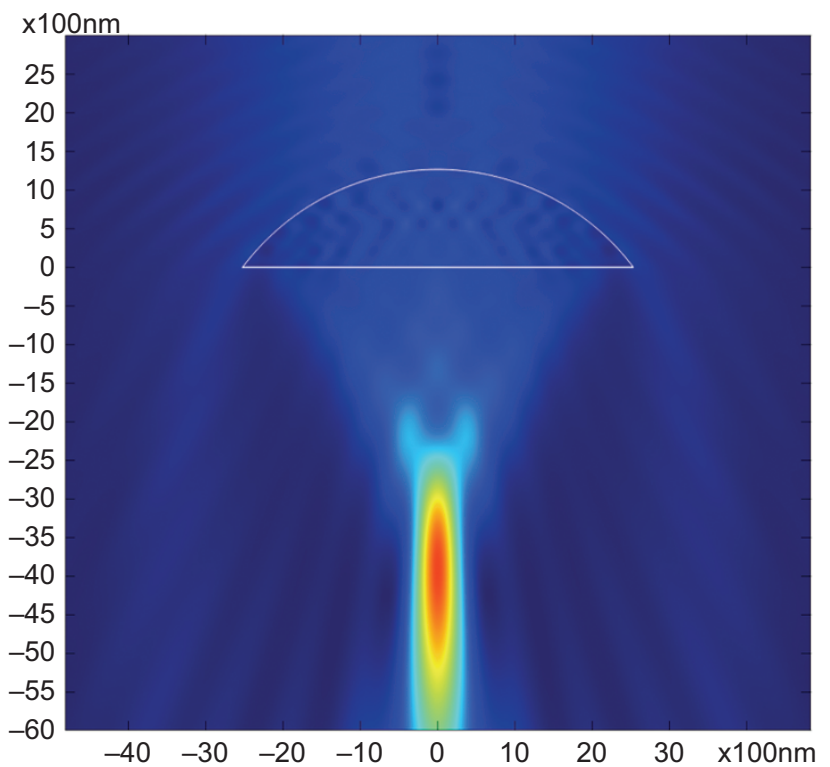

b

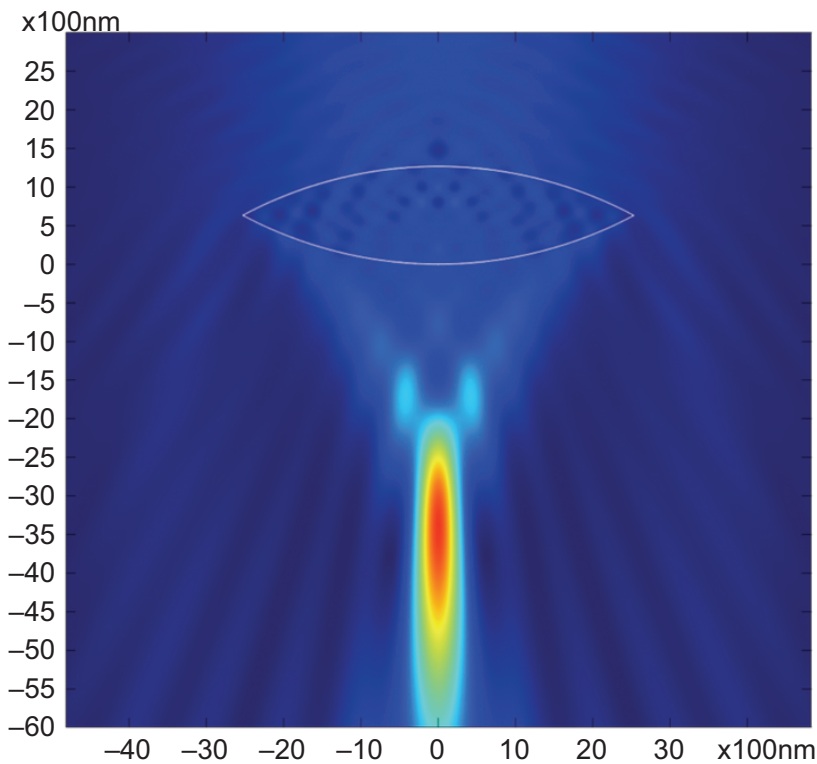

d

$\mathrm{x} 100 \mathrm{~nm}$

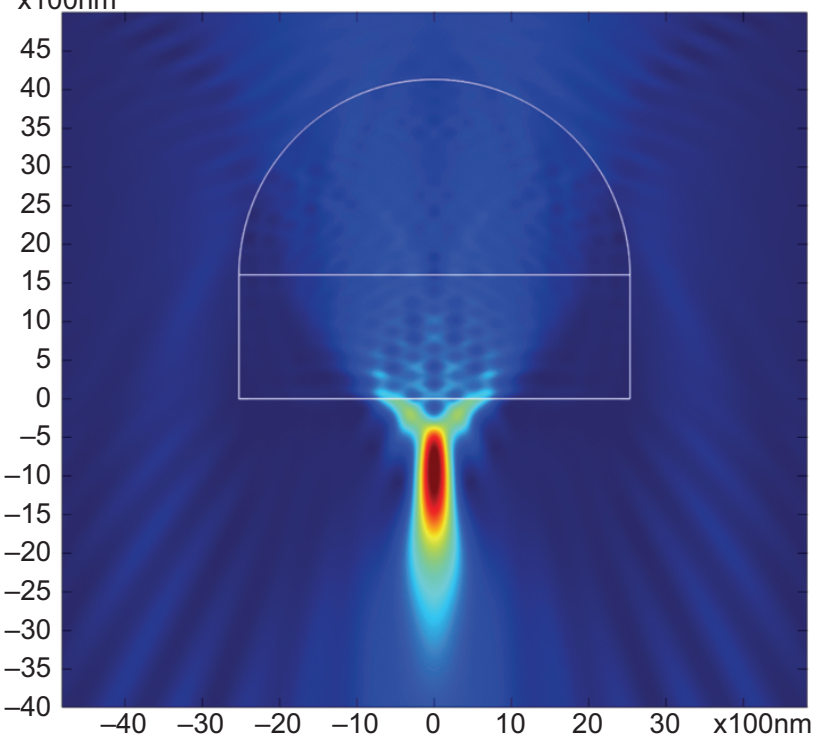

$\begin{array}{lllllll}1 & 2 & 3 & 4 & 5 & 6 & 7\end{array}$

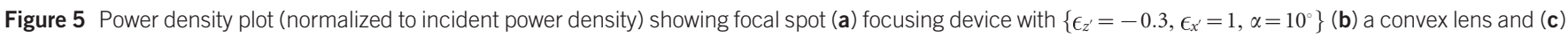

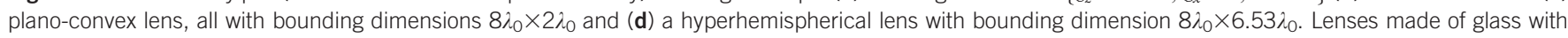
$\epsilon_{r}=2.5$. The incident Gaussian beam has a waist size of $\omega_{0}=6 \lambda_{0}$.

There are several parameters that determine the operation of the device, primarily the effective permittivity $\left\{\epsilon_{z^{\prime}}, \epsilon_{x^{\prime}}\right\}$, the tilt angle $\alpha$ and the thickness. These parameters determine the location of the focal spot with respect to the exit facet as well as the strength of the focus. For an ideal device with parameters $\left\{\epsilon_{z^{\prime}}=-0.14, \epsilon_{x^{\prime}}=\right.$ $\left.1.068, \alpha=10^{\circ}\right\}$ and a fixed aperture size of $8 \lambda_{0}$, Figure 6 a shows the change in the focal spot location as a function of the device thickness, as well as the strength of the focus sensed. The results show that thinner devices (even thinner than one wavelength) are also possible yielding light concentration at extremely short distances. Very thin devices come at the expense of a reduced focal strength. Given the observed trends for focal strength and location, one may conclude that a good range for the thickness is $\lambda_{0} / 2-3 \lambda_{0} / 2$, which provides $5-11$ times power intensification of the incident power density. For instance, a thin device with thickness of only $\lambda_{0}$ yields a short focal distance of $0.6 \lambda_{0}$ and a focal strength intensification of 10 times (an order of magnitude) higher than the incident power density. Such device outperforms Fresnel lenses in terms of size, as they are normally in the 
a

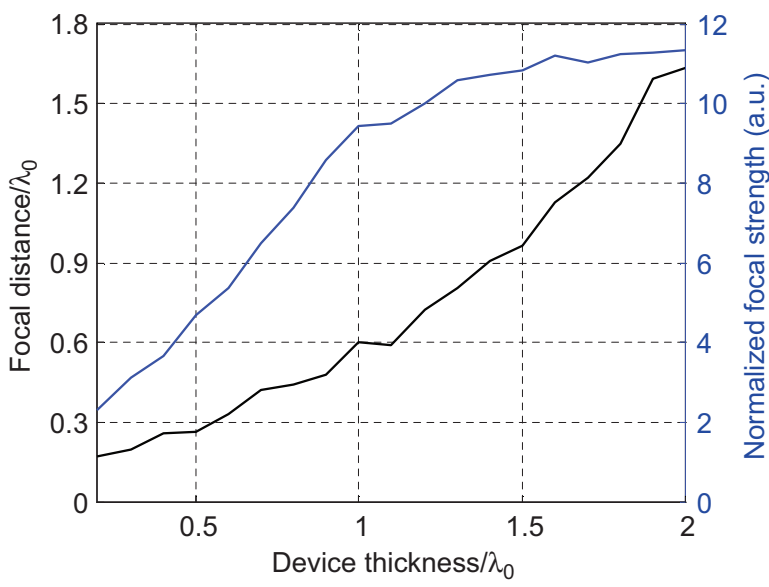

b

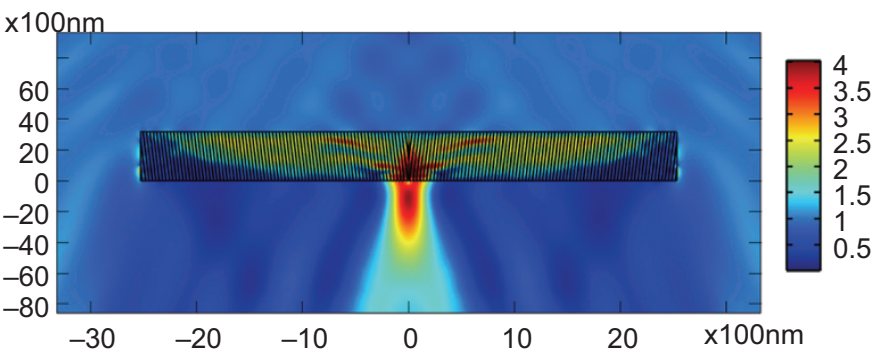

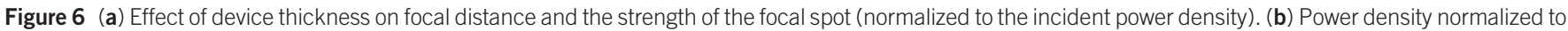
incident power density for a lossy focusing device that is $\lambda_{0} / 2$ in height and $8 \lambda_{0}$ wide, realized with bilayers of Ag/glass.

order of several wavelengths. ${ }^{40}$ The $f / D$ ratio for the range of devices in Figure $6 \mathrm{a}$ is $0.02-0.2$, for instance, $f / D=0.075$ for the $\lambda_{0}$ thick case.

Figure $6 \mathrm{~b}$ shows a half-wavelength thick lossy focusing device realized with Ag/glass, which is still able to achieve concentration at a distance of about $\lambda_{0} / 4$. Note that the low effective longitudinal permittivity and the unique refraction are enabling this very thin design, as the refracted waves have a very short longitudinal path inside the structure as discussed earlier. With a layered realization, the permittivity of the focusing device is adjustable by tuning the filling ratio using the same materials, e.g., Ag and glass used here. This is while a lens permittivity can only be changed by using a different material to increase the refractive index. In addition to these extremely thin designs, the device may also be scaled to electrically large aperture size lenses by adjusting its thickness accordingly.

The lensing effect occurs for any wavelength in the theoretical device as long as the effective longitudinal permittivity, $\epsilon_{z^{\prime}}$, is within an acceptable limit. Therefore, the principle of operation is potentially wideband. However, depending on the implementation (e.g., whether bilayered, nanowire media, nanocomposite, etc.), the bandwidth limits vary accordingly. The bandwidth of the bilayered implementation primarily depends on the choice of the metal and its frequency dispersion characteristics. As the frequency changes, the negative permittivity of the metal varies according to its dispersion; hence, the effective $\epsilon_{z^{\prime}}$ varies. Some of the overall effects of varying the operating frequency are changes in the focus intensity and a slight reduction of the focal distance from the exit facet of the device. For the specific Ag/ glass example presented in Figure 4, our simulations indicate that the lens has an operating line-width of $200 \mathrm{~nm}$ ranging from 590 to $790 \mathrm{~nm}$. This is based on a stringent condition requiring that a distinct focus with at least $2 \times$ power intensification is achieved at the band edges.

\section{CONCLUSIONS}

A flat low-profile focusing device is presented, using a hetero-junction of anisotropic low-permittivity MTMs. These MTMs guide the wave away from normal and along the interface, allowing for a thin realization. The proposed focusing hetero-junctions are realizable at optical frequencies using periodic bilayers of metal and dielectric. The proposed device can focus an incoming plane or Gaussian wave into an area of high power concentration, at a distance much closer than lenses with low $f / D$ ratios for the same size requirements, as well as the recently reported arrays of optical antennas and Fresnel lenses. The proposed hetero-junction may be used in the low-loss focusing mode for applications requiring light concentration such as concentrated photovoltaics, non-imaging optics, micro- and nano-Fresnel lenses, photodetectors, etc., as well as in the high-loss mode for realizing thin absorbers/emitters for solar and/or thermo photovoltaics.

\section{ACKNOWLEDGMENTS}

This work was supported by the Natural Sciences and Engineering Research Council of Canada and by Defence Research and Development Canada.

1 Winston R, Minano JC, Welford WT, Benitez P. Nonimaging Optics. San Diego, CA: Academic Press; 2004.

2 Karp JH, Tremblay EJ, Ford JE. Planar micro-optic solar concentrator. Opt Express 2010; 18: 1122-1133.

3 Xie WT, Dai YJ, Wang RZ, Sumathy K. Concentrated solar energy applications using Fresnel lenses: a review. Renew Sustain Energy Rev 2011; 15: 2588-2606.

4 Rahm M, Schurig D, Roberts DA, Cummer SA, Smith DR et al. Design of electromagnetic cloaks and concentrators using form-invariant coordinate transformations of Maxwell's equations. Photon Nanostruct Fundam App/ 2008; 6: 87-95.

5 Wang W, Lin L, Ma J, Wang C, Cui J et al. Electromagnetic concentrators with reduced material parameters based on coordinate transformation. Opt Express 2008; 16: 11431-11437.

6 Yang J, Huang M, Yang C, Xiao Z, Peng J. Metamaterial electromagnetic concentrators with arbitrary geometries. Opt Express 2009; 17: 19656-19661.

7 Jiang WX, Chin JY, Cui TJ. Anisotropic metamaterial devices. Mater Today 2009; 12 : 26-33.

8 Pendry JB, Schurig D, Smith DR. Controlling electromagnetic fields. Science 2006; 312: 1780-1782.

9 Pendry JB. Negative refraction makes a perfect lens. Phys Rev Lett 2000; 85: 3966 3969.

10 Smith DR, Padilla WJ, Vier DC, Nemat-Nasser SC, Schultz S. Composite medium with simultaneously negative permeability and permittivity. Phys Rev Lett 2000; 84 : 4184-4187.

11 Grbic A, Eleftheriades GV. Overcoming the diffraction limit with a planar left-handed transmission-line lens. Phys Rev Lett 2004; 92: 117403.

12 Morgado TA, Marcos JS, Maslovski SI, Silveirinha MG. Negative refraction and partial focusing with a crossed wire mesh: physical insights and experimental verification. Appl Phys Lett 2012; 101: 021104.

13 Smith DR, Schurig D. Electromagnetic wave propagation in media with indefinite permittivity and permeability tensors. Phys Rev Lett 2003; 90: 077405.

14 Kaipa CSR, Yakovlev AB, Maslovski SI, Silveirinha MG. Near-field imaging with a loaded wire medium. Phys Rev B 2012; 86: 155103.

15 Cui Y, Fung KH, Xu J, Ma H, Jin Y et al. Ultrabroadband light absorption by a sawtooth anisotropic metamaterial slab. Nano Lett 2012; 12: 1443-1447. 
16 Avitzour Y, Urzhumov YA, Shvets G. Wide-angle infrared absorber based on a negativeindex plasmonic metamaterial. Phys Rev B 2009; 79: 045131.

17 Rephaeli E, Fan S. Tungsten black absorber for solar light with wide angular operation range. Appl Phys Lett 2008; 92: 211107.

18 Sergeant NP, Agrawal M, Peumans P. High performance solar-selective absorbers using coated sub-wavelength gratings. Opt Express 2010; 18: 5525-5540.

19 Hao J, Wang J, Liu X, Padilla WJ, Zhou L et al. High performance optical absorber based on a plasmonic metamaterial. Appl Phys Lett 2010; 96: 251104.

20 Wu C, Neuner B, Shvets G, John J, Milder A et al. Large-area wide-angle spectrally selective plasmonic absorber. Phys Rev B 2011; 84: 075102.

21 Nefzaoui E, Drevillon J, Joulain K. Selective emitters design and optimization for thermophotovoltaic applications. J App/ Phys 2012; 111: 084316.

22 Liu X, Tyler T, Starr T, Starr AF, Jokerst NM et al. Taming the blackbody with infrared metamaterials as selective thermal emitters. Phys Rev Lett 2011; 107: 045901.

23 D'Aguanno G, Mattiucci N, Alu A, Argyropoulos C, Foreman JV et al. Thermal emission from a metamaterial wire medium slab. Opt Express 2012; 20: 9784-9789.

24 Mattiucci N, D'Aguanno G, Alu A, Argyropoulos C, Foreman JV et al. Taming the thermal emissivity of metals: a metamaterial approach. App Phys Lett 2012; 100 201109.

25 Mason JA, Smith S, Wasserman D. Strong absorption and selective thermal emission from midinfrared metamaterials. App Phys Lett 2011; 98: 241105.

26 Bermel P, Ghebrebrhan M, Harradon M, Yeng YX, Celanovic I et al. Tailoring photonic metamaterial resonances for thermal radiation. Nanoscale Res Lett 2011; 6: 549

27 Wu C, Neuner B, John J, Milder A, Zollars B et al. Metamaterial-based integrated plasmonic absorber/emitter for solar thermo-photovoltaic systems. J Opt 2012; 14 024005.

28 Rephaeli E, Fan S. Absorber and emitter for solar thermophotovoltaic systems to achieve efficiency exceeding the Shockley-Queisser limit. Opt Express 2009; 17: 145-159.

29 Engheta N. Pursuing near-zero response. Science 2013; 340: 286-287.

30 Silveirinha M, Engheta N. Tunneling of electromagnetic energy through subwavelength channels and bends using $\epsilon$-near-zero materials. Phys Rev Let 2006; 97: 157403.
31 Alu A, Silveirinha MG, Salandrino A, Engheta N. Epsilon-near-zero metamaterials and electromagnetic sources: tailoring the radiation phase pattern. Phys Rev B 2007; 75: 155410.

32 Collin RE. A simple artificial anisotropic dielectric medium. IRE Trans Microw Theory Tech 1958; 6: 206-209.

33 Fang A, Koschny T, Soukoulis CM. Optical anisotropic metamaterials: negative refraction and focusing. Phys Rev B 2009; 79: 245127.

34 Liu Y, Bartal G, Zhang X. All-angle negative refraction and imaging in a bulk medium made of metallic nanowires in the visible region. Opt Express 2008; 16: 1543915448 .

35 Salandrino A, Engheta N. Far-field subdiffraction optical microscopy using metamaterial crystals: theory and simulations. Phys Rev B 2006; 74: 075103.

36 Jacob Z, Alekseyev L, Narimanov E. Optical hyperlens: far-field imaging beyond the diffraction limit. Opt Express 2006; 14: 8247-8256.

37 Liu Z, Lee H, Xiong Y, Sun C, Zhang X. Far-field optical hyperlens magnifying subdiffraction-limited objects. Science 2007; 315: 1686.

38 Lee H, Liu Z, Xiong Y, Sun C, Zhang X. Development of optical hyperlens for imaging below the diffraction limit. Opt Express 2007; 15: 15886-15891.

39 Aieta F, Genevet P, Kats MA, Yu N, Blanchard R et al. Aberration-free ultrathin flat lenses and axicons at telecom wavelengths based on plasmonic metasurfaces. Nano Lett 2012; 12: 4932-4936.

$40 \mathrm{NiX}$, Ishii S, Kildishev AV, Shalaev VM. Ultra-thin, planar, Babinet-inverted plasmonic metalenses. Light Sci App/2013; 2: e72; doi:10.1038/lsa.2013.28.

41 Boltasseva A, Atwater HA. Low-loss plasmonic metamaterials. Science 2011; 331: 290-291.

42 Filipovic DF, Gearhart SS, Rebeiz GM. Double-slot antennas on extended hemispherical and elliptical silicon dielectric lenses. IEEE Trans Microw Theory Tech 1993; 41: 1738-1749.

\section{(c) (i) ()}

This work is licensed under a Creative Commons AttributionNonCommercial-ShareAlike 3.0 Unported license. To view a copy of this license, visit http://creativecommons.org/licenses/by-nc-sa/3.0 